\title{
Factorization of unbounded operators on Köthe spaces
}

\author{
by \\ T. TerzioĞLu (Istanbul), M. Yurdakul (Ankara) \\ and V. ZAHARIUTA (Istanbul)
}

\begin{abstract}
The main result is that the existence of an unbounded continuous linear operator $T$ between Köthe spaces $\lambda(A)$ and $\lambda(C)$ which factors through a third Köthe space $\lambda(B)$ causes the existence of an unbounded continuous quasidiagonal operator from $\lambda(A)$ into $\lambda(C)$ factoring through $\lambda(B)$ as a product of two continuous quasidiagonal operators. This fact is a factorized analogue of the Dragilev theorem $[3,6,7,2]$ about the quasidiagonal characterization of the relation $(\lambda(A), \lambda(B)) \in \mathcal{B}$ (which means that all continuous linear operators from $\lambda(A)$ to $\lambda(B)$ are bounded). The proof is based on the results of [9] where the bounded factorization property $\mathcal{B F}$ is characterized in the spirit of Vogt's [10] characterization of $\mathcal{B}$. As an application, it is shown that the existence of an unbounded factorized operator for a triple of Köthe spaces, under some additonal asumptions, causes the existence of a common basic subspace at least for two of the spaces (this is a factorized analogue of the results for pairs $[8,2]$ ).
\end{abstract}

1. Introduction. We denote by $\lambda(A)$ the Köthe space defined by the matrix $A=\left(a_{i}^{p}\right)$, and by $\left(e_{n}\right)$ the canonical basis of $\lambda(A)$. For a mapping $\sigma: \mathbb{N} \rightarrow \mathbb{N}$ and a sequence $\left(t_{n}\right)$ of scalars the operator $D: \lambda(A) \rightarrow \lambda(B)$ defined by $D\left(e_{n}\right)=t_{n} e_{\sigma(n)}, n \in \mathbb{N}$, is called quasidiagonal. Dragilev [3] proved that the existence of an unbounded continuous linear operator from $\lambda(A)$ to $\lambda(B)$, where both spaces are assumed to be nuclear, implies the existence of a continuous unbounded quasidiagonal operator from $\lambda(A)$ to $\lambda(B)($ cf. $[6,7])$. This result has recently been generalized by Djakov and Ramanujan [2] by omitting the nuclearity assumption.

We recall that the closed linear span of a subbasis $\left(e_{i_{n}}\right)$ is called a basic subspace of a Köthe space. If $\lambda(A)$ and $\lambda(B)$ have a common basic subspace, then it is easy to construct a continuous linear operator mapping $\lambda(A)$ into $\lambda(B)$, which is unbounded unless the common basic subspace is a Banach space. Under certain conditions on $\lambda(A)$ and $\lambda(B)$ the converse of this trivial fact is also true. Namely, if both spaces are nuclear, Nurlu and Terzioğlu [8]

2000 Mathematics Subject Classification: 46A03, 46A13, 46A04, 46A20, 46A32, 47L05.

Key words and phrases: locally convex spaces, unbounded operators, Köthe spaces, bounded factorization property. 
proved that the existence of an unbounded continuous linear operator $T$ : $\lambda(A) \rightarrow \lambda(B)$ implies, under some additional conditions, the existence of a common basic subspace of $\lambda(A)$ and $\lambda(B)$; this result was generalized by Djakov and Ramanujan in [2] to the non-nuclear case. In these works Dragilev's theorem plays a crucial role.

It was discovered in $[13,14]$ that if the matrices $A$ and $B$ satisfy the conditions $d_{2}, d_{1}$, respectively, then every continuous linear operator from $\lambda(A)$ into $\lambda(B)$ is bounded. This phenomenon was studied extensively by many authors; the most comprehensive result is due to Vogt [10], where all pairs of Fréchet spaces with this property are characterized. Terzioğlu and Zahariuta [9] characterized those triples $(X, Y, Z)$ of Fréchet spaces such that each continuous linear operator $T: X \rightarrow Z$ which factors through $Y$ is automatically bounded.

The aim of the present work is to prove a factorization analogue of Dragilev's theorem [3] and its generalization [2]. Namely, we prove that if there is an unbounded continuous linear operator $T: \lambda(A) \rightarrow \lambda(C)$ which factors through $\lambda(B)$, then, in fact, there exists an unbounded continuous quasidiagonal operator $D: \lambda(A) \rightarrow \lambda(C)$ that factors through $\lambda(B)$ as a product of two continuous quasidiagonal operators. As an application, similarly to $[8,2]$, we show that the existence of an unbounded factorized operator for a triple of Köthe spaces causes that, under some additional conditions, these spaces (or at least two of them) have a common basic subspace.

\section{Bounded factorization property and quasidiagonal operators.} We denote by $L(X, Y)$ and $L B(X, Y)$ the spaces of all continuous linear operators and of all bounded linear operators from the locally convex space $X$ into the locally convex space $Y$. If for each $S \in L(X, Y)$ and $R \in L(Y, Z)$ we have $T=R S \in L B(X, Z)$, we say $(X, Y, Z)$ has the bounded factorization property and write $(X, Y, Z) \in \mathcal{B F}([9])$.

Dealing with several Fréchet spaces we always use the same notation $\left\{|\cdot|_{p}: p \in \mathbb{N}\right\}$ for a system of seminorms defining their topologies and $\left\{|\cdot|_{p}^{*}: p \in \mathbb{N}\right\}$ for the corresponding system of polar norms in the dual spaces. For any operator $T \in L(E, F)$ we consider the operator seminorms

$$
|T|_{p, q}=\sup \left\{|T x|_{p}:|x|_{q} \leq 1\right\}, \quad p, q \in \mathbb{N},
$$

which may take the value $+\infty$. In particular, for any one-dimensional operator $T=x^{\prime} \otimes y, x^{\prime} \in E^{\prime}, y \in F$, we have $|T|_{p, q}=\left|x^{\prime}\right|_{q}^{*} \cdot|y|_{p}$.

Dealing with a Köthe space $\lambda(A)$ we always assume that the matrix $A=\left(a_{i}^{p}\right)$ satisfies the condition

$$
a_{i}^{p} \leq a_{i}^{p+1}, \quad i, p \in \mathbb{N} .
$$

An operator $T \in L(\lambda(A), \lambda(B))$ is quasidiagonal if $T\left(e_{i}\right)=t_{i} e_{\tau(i)}, i \in \mathbb{N}$, for 
some map $\tau: \mathbb{N} \rightarrow \mathbb{N}$ and scalar sequence $\left(t_{i}\right)$. We denote by $Q(A, B)$ the set of all quasidiagonal operators and by $Q_{\tau}(A, B)$ its subset corresponding to the map $\tau$. We note that $Q_{\tau}(A, B)$ is a subspace of $L(\lambda(A), \lambda(B))$ whereas $Q(A, B)$ is only a subset.

Our aim is to prove the following characterization of the bounded factorization property for triples of Köthe spaces in terms of quasidiagonal operators, which is a natural generalization of Dragilev's theorem $([3,2])$.

THEOREM 1. We have $(\lambda(A), \lambda(B), \lambda(C)) \in \mathcal{B F}$ if and only if for each $S \in Q(A, B)$ and $R \in Q(B, C)$ the quasidiagonal operator $T=R S$ is bounded.

The proof will be given in Section 3 after some intermediate results. In what follows we will use the following result from [9].

Proposition 2. We have $(\lambda(A), \lambda(B), \lambda(C)) \in \mathcal{B} \mathcal{F}$ if and only if for each non-decreasing map $\pi: \mathbb{N} \rightarrow \mathbb{N}$ there is $r \in \mathbb{N}$ such that for every $q \in \mathbb{N}$ there exists $n=n(q) \in \mathbb{N}$ so that the inequality

$$
\frac{c_{i}^{q}}{a_{j}^{r}} \leq n \max _{p=1, \ldots, n}\left\{\frac{b_{\nu}^{p}}{a_{j}^{\pi(p)}}\right\} \cdot \max _{p=1, \ldots, n}\left\{\frac{c_{i}^{p}}{b_{\nu}^{\pi(p)}}\right\}
$$

holds for all $(i, j, \nu) \in \mathbb{N}^{3}$.

Given two Fréchet spaces $E$ and $F$ and a map $\pi: \mathbb{N} \rightarrow \mathbb{N}$, we consider the Fréchet space

$$
L_{\pi}(E, F):=\left\{T \in L(E, F):|T|_{p, \pi(p)}<\infty, p \in \mathbb{N}\right\}
$$

with the topology generated by the system of seminorms $\left\{|\cdot|_{p, \pi(p)}: p \in \mathbb{N}\right\}$.

We note that, in the case of Köthe spaces, the intersection

$$
Q_{\sigma}^{\pi}(A, B):=Q_{\sigma}(A, B) \cap L_{\pi}(\lambda(A), \lambda(B))
$$

is a closed subspace of $L_{\pi}(\lambda(A), \lambda(B))$. Fix $\sigma, \varrho$, and $\pi$ and assume that for each $S \in L_{\sigma}(A, B), R \in L_{\varrho}(B, C)$ the composition $R S$ is bounded. If we apply Lemma 2.1 from [9] to the bilinear map

$$
\theta: Q_{\sigma}^{\pi}(A, B) \times Q_{\varrho}^{\pi}(B, C) \rightarrow L B(\lambda(A), \lambda(C))
$$

which simply sends each $(S, R)$ to $R S$, we obtain the following result.

Proposition 3. Let $\sigma$ and $\varrho$ be two maps of $\mathbb{N}$ into $\mathbb{N}$. If for each $S \in Q_{\sigma}(A, B)$ and $R \in Q_{\varrho}(B, C)$ the composition $R S$ is bounded, then for each $\pi: \mathbb{N} \rightarrow \mathbb{N}$ there is $r \in \mathbb{N}$ such that for every $q \in \mathbb{N}$ there exists $n=n(q) \in \mathbb{N}$ such that the inequality

$$
\frac{c_{\varrho(\sigma(j))}^{q}}{a_{j}^{r}} \leq n \max _{p=1, \ldots, n}\left\{\frac{b_{\sigma(j)}^{p}}{a_{j}^{\pi(p)}}\right\} \cdot \max _{p=1, \ldots, n}\left\{\frac{c_{\varrho(\sigma(j))}^{p}}{b_{\sigma(j)}^{\pi(p)}}\right\}
$$

holds for every $j \in \mathbb{N}$. 
We note that here both $r$ and $n$ depend not only on $\pi$ and $q$ but also on our choice of $\sigma$ and $\varrho$. This is an obstacle to deriving Theorem 1 immediately from Proposition 3. On the other hand, the methods of [9] cannot be applied directly to $Q(A, B)$, since it is not a subspace. So we need some other considerations.

3. Proof of Theorem 1. Suppose $(\lambda(A), \lambda(B), \lambda(C)) \notin \mathcal{B F}$. Then, by Proposition 2, there is a non-decreasing map $\pi: \mathbb{N} \rightarrow \mathbb{N}$ such that for each $r \in \mathbb{N}$ there exists $q=q(r) \in \mathbb{N}$ such that for any $n \in \mathbb{N}$ there are $i_{n}=i_{n}(r)$, $j_{n}=j_{n}(r), \nu_{n}=\nu_{n}(r)$ with

$$
\frac{c_{i_{n}}^{q}}{a_{j_{n}}^{r}}>n \max _{p=1, \ldots, n}\left\{\frac{b_{\nu_{n}}^{p}}{a_{j_{n}}^{\pi(p)}}\right\} \cdot \max _{p=1, \ldots, n}\left\{\frac{c_{i_{n}}^{p}}{b_{\nu_{n}}^{\pi(p)}}\right\} .
$$

With this notation we have the following technical result, which is crucial for our proof.

Lemma 4. For any $r \geq r_{0}=\pi(\pi(1))$ the sequences $\left(i_{n}\right)_{n},\left(j_{n}\right)_{n},\left(\nu_{n}\right)_{n}$ diverge to $+\infty$.

Proof. First we notice that (4) is equivalent to the system of inequalities

$$
\frac{c_{i_{n}}^{q}}{a_{j_{n}}^{r}}>n \frac{b_{\nu_{n}}^{p} \cdot c_{i_{n}}^{s}}{a_{j_{n}}^{\pi(p)} \cdot b_{\nu_{n}}^{\pi(s)}}, \quad 1 \leq p, s \leq n .
$$

Suppose that $j_{n}$ does not tend to $+\infty$, that is, $j_{n_{k}}=j=$ const for some subsequence $n_{k}$. This contradicts (5): take $s=q, p=\pi(q), n=n_{k}>\pi(q)$.

Analogously, assuming that $i_{n_{k}}=i=$ const for some subsequence $n_{k}$, we get a contradiction by putting $s=1, p=\pi(1), n=n_{k}>\pi(1)$ in (5) and taking into account the assumption $r \geq \pi(\pi(1))$.

Finally, the assumption $\nu_{n_{k}}=\nu=$ const also leads to a contradiction: consider (5) with $s=q, p=1, n=n_{k}>q$, remembering that $r \geq r_{0}$ $\geq \pi(1)$.

We are now ready to prove a result which is somewhat stronger than Theorem 1.

Proposition 5. If $(\lambda(A), \lambda(B), \lambda(C)) \notin \mathcal{B F}$ then there are bijections $\sigma$ and $\varrho$ on $\mathbb{N}$ and operators $S \in Q_{\sigma}(A, B)$ and $R \in Q_{\varrho}(B, C)$ such that the operator $T=R S$ is unbounded.

Proof. From our assumption we have (4) with the same notation. Passing to subsequences three times and using Lemma 4 , for any fixed $r \geq r_{0}:=$ $\pi(\pi(1))$ we construct a subsequence $L_{r}=\left\{n_{k}(r)\right\}$ of $\mathbb{N}$ such that each coordinate of $\left(j_{n_{k}(r)}, \nu_{n_{k}(r)}, i_{n_{k}(r)}\right)$ takes different values for different $k$. Let us 
represent each infinite set $L_{r}$ as a disjoint union of infinite subsets

$$
L_{r}=\bigcup_{\mu=0}^{\infty} L_{r, \mu} .
$$

Let us now construct a new sequence of infinite disjoint sets

$$
\widetilde{L}_{r}=\left\{l_{\mu}(r): \mu \in \mathbb{N}\right\} \subset L_{r}, \quad r \geq r_{0},
$$

in the following inductive way. We form $\widetilde{L}_{r_{0}}$ by taking precisely one element $l_{\mu}\left(r_{0}\right)$ from each $L_{r_{0}, \mu}, \mu \in \mathbb{N}$. Assume we have already constructed pairwise disjoint sets $\widetilde{L}_{s}$ for $r_{0} \leq s \leq r$, so that each $\widetilde{L}_{s}$ contains exactly one element from $L_{s, \mu}$ and is disjoint from $L_{s, 0}$. We then construct $\widetilde{L}_{r+1}$ by taking from each $L_{r+1, \mu}, \mu \in \mathbb{N}$, one element different from every $l_{\mu}(s), r_{0} \leq s \leq r$. By induction this concludes the construction of $\widetilde{L}_{r}, r \geq r_{0}$. The set $I_{0}:=$ $\mathbb{N} \backslash \bigcup_{r=r_{0}}^{\infty} I_{\widetilde{L}_{r}}$ is infinite since it contains $I_{L_{r, 0}}$ for each $r \geq r_{0}$. By the same token the sets

$$
J_{0}:=\mathbb{N} \backslash \bigcup_{r=r_{0}}^{\infty} J_{\widetilde{L}_{r}}, \quad N_{0}:=\mathbb{N} \backslash \bigcup_{r=r_{0}}^{\infty} N_{\widetilde{L}_{r}}
$$

are also infinite.

Let $\alpha: J_{0} \rightarrow N_{0}$ and $\beta: N_{0} \rightarrow I_{0}$ be arbitrary bijections. Consider the maps $\varrho: \mathbb{N} \rightarrow \mathbb{N}$ and $\sigma: \mathbb{N} \rightarrow \mathbb{N}$ defined by

$$
\begin{aligned}
& \sigma(j):= \begin{cases}\alpha(j) & \text { if } j \in J_{0}, \\
\nu_{l_{\mu}(r)} & \text { if } j=j_{l_{\mu}(r)} \in J_{\widetilde{L}_{r}}, r \geq r_{0},\end{cases} \\
& \varrho(\nu):= \begin{cases}\beta(\nu) & \text { if } \nu \in N_{0}, \\
i_{l_{\mu}(r)} & \text { if } \nu=\nu_{l_{\mu}(r)} \in N_{\widetilde{L}_{r}}, r \geq r_{0} .\end{cases}
\end{aligned}
$$

For each $r$ we have

$$
\frac{c_{\varrho(\sigma(j))}^{q(r)}}{a_{j}^{r}}>n \max _{p=1, \ldots, n}\left\{\frac{b_{\sigma(j)}^{p}}{a_{j}^{\pi(p)}}\right\} \cdot \max _{p=1, \ldots, n}\left\{\frac{c_{\varrho(\sigma(j))}^{p}}{b_{\sigma(j)}^{\pi(p)}}\right\}
$$

for all $j=j_{n}$, where $n \in \widetilde{L}_{r}$. Hence by Proposition 3, there exist $S \in$ $Q_{\sigma}(A, B)$ and $R \in Q_{\varrho}(B, C)$ with $R S$ unbounded.

4. Some consequences. Nurlu and Terzioğlu [8] studied the consequences of the existence of an unbounded operator between nuclear Köthe spaces. They showed, in particular, that if the spaces satisfy a splitting condition of Apiola type [1], then the existence of an unbounded operator implies the existence of a common basic subspace. Djakov and Ramanujan [2] obtain the same result without the assumption of nuclearity and assuming the weaker splitting condition of Krone and Vogt [5]. 
Before dealing with the main result of this section (see Theorem 10 below) we discuss certain modifications and factorized analogues of some properties, important for studying the relation $\operatorname{Ext}^{1}(F, E)=0$ (see, e.g., $[11,12,4])$. A pair $(F, E)$ of Fréchet spaces satisfies the condition $\mathcal{S}$ if there is a mapping $\tau: \mathbb{N} \rightarrow \mathbb{N}$ such that for all $p, r \in \mathbb{N}$ there exists a constant $C=C(p, r)$ such that the estimate

$$
|T|_{r, \tau(p)} \leq C \max \left\{|T|_{\tau(p), p},|T|_{\tau(r), r}\right\}
$$

holds for any one-dimensional operator

$$
T=e^{\prime} \otimes f, \quad e^{\prime} \in E^{\prime}, f \in F .
$$

It is easy to check that the condition $\mathcal{S}$ is an equivalent slight variation of Vogt's condition $S_{2}^{*}([11])$. It is known that the property $\operatorname{Ext}^{1}(F, E)=0$ is characterized by $(F, E) \in \mathcal{S}$ whenever both spaces are either Köthe spaces ([5]) or nuclear ([4]). A pair of Köthe spaces $E=\lambda(A)$ and $F=\lambda(B)$ satisfies the condition $\mathcal{S}$ if and only if the condition (6) holds for the operators $T=e_{i}^{\prime} \otimes e_{j}, i, j \in \mathbb{N}([5])$.

If the estimate (6) is true for arbitrary operators $T \in L(E, F)$ (with an obvious meaning if some of the operator norms equals $+\infty$ ) then we write $(F, E) \in \overline{\mathcal{S}}$ (in fact, one can see that this condition is reasonable only for bounded operators $T)$. It is easy to check that the condition $(F, E) \in \overline{\mathcal{S}}$ coincides with the condition on $L B(E, F)$ considered by Dierolf, Frerick, Mangino, and Wengenroth (see, e.g., [4, the proof of Theorem 2.2]); moreover, by Vogt [12], this condition coincides with the condition $(w Q)$ for the natural representation of $L B(E, F)$ as an $(L F)$-space.

In what follows we shall denote by $\lambda(A)_{L}$ the basic subspace of a Köthe space $\lambda(A)$ which is the closed linear envelope of $\left\{e_{n}: n \in L\right\}, L \subset \mathbb{N}$.

Suppose now $(\lambda(A), \lambda(B), \lambda(C)) \notin \mathcal{B F}$ and $(\lambda(C), \lambda(A)) \in \mathcal{S}$. By Theorem 1 we know that there are $S \in Q_{\sigma}(A, B)$ and $R \in Q_{\varrho}(B, C)$ with some bijective maps $\sigma$ and $\varrho$ on $\mathbb{N}$ such that $T=R S$ is an unbounded quasidiagonal operator. The theorem of Djakov and Ramanujan [2] implies the existence of infinite subsets $J$ and $I$ of $\mathbb{N}$ such that $T$ maps $\lambda(A)_{J}$ isomorphically onto $\lambda(C)_{I}$. Then one can easily check that for $N:=\sigma(J)=\varrho^{-1}(I)$ both $S: \lambda(A)_{J} \rightarrow \lambda(B)_{N}$ and $R: \lambda(B)_{N} \rightarrow \lambda(C)_{J}$ are also isomorphisms. We have therefore proved the following result.

Proposition 6. Let $E=\lambda(A), G=\lambda(B)$, and $F=\lambda(C)$. Suppose that $(E, G, F) \notin \mathcal{B F}$ and $(F, E) \in \mathcal{S}$. Then there is a common basic subspace for all three spaces.

Now we consider a factorized analogue of the condition $\mathcal{S}$. A triple of Fréchet spaces $(F, G, E)$ satisfies the condition $\mathcal{S F}$ (we write $(F, G, E) \in$ $\mathcal{S F}$ ) if for any one-dimensional operator $T=R S$, with both $S \in L(E, G)$ 
and $R \in L(G, F)$ also one-dimensional, the inequality

$$
|T|_{r, \tau(p)} \leq C \max \left\{|R|_{\tau(p), p},|R|_{\tau(r), r}\right\} \cdot \max \left\{|S|_{\tau(p), p},|S|_{\tau(r), r}\right\}
$$

holds with the same requisites as in (6).

If the condition (7) holds for an arbitrary operator $T=R S$ with $S \in$ $L(E, G)$ and $R \in L(G, F)$ we will write $(F, G, E) \in(\overline{\mathcal{S F}})$ (with the evident meaning when some of the operator norms equals $+\infty$; in fact, this condition is reasonable only for bounded operators $T$ ).

We note that if $E=G$ or $G=F$ the condition $(F, G, E) \in \mathcal{S F}$ reduces simply to $(F, E) \in \mathcal{S}$, and $(F, G, E) \in \overline{\mathcal{S F}}$ reduces to $(F, E) \in \overline{\mathcal{S}}$.

Proposition 7. Let $E, G, F$ be arbitrary Fréchet spaces. If $(E, G, F)$ $\in \mathcal{B F}$, then $(F, G, E) \in \overline{\mathcal{S F}}$.

Proof. Suppose that $(E, G, F) \in \mathcal{B F}$. Denote by $\Pi(p)$ the set of all strictly increasing mappings $\pi \in \mathbb{N}^{\mathbb{N}}$ such that $\pi(1)=p$. By Theorem 2.2 from [9], for any $\pi \in \Pi(p)$ there are $q \in \mathbb{N}$ and $\mu \in \mathbb{N}^{\mathbb{N}}$ such that for every $T=R S$ with $S \in L(E, G)$ and $R \in L(G, F)$ the inequality

$$
|T|_{r, q} \leq \mu(r) \max _{l=1}^{\mu(r)}\left\{|R|_{l, \pi(l)}\right\} \cdot \max _{l=1}^{\mu(r)}\left\{|S|_{l, \pi(l)}\right\}
$$

holds for each $r \in \mathbb{N}$. We denote by $\Pi_{q}(p)$ the set of all $\pi \in \Pi(p)$ satisfying (8) with a given $q \in \mathbb{N}$. It is obvious that $\Pi(p)=\bigcup_{q=1}^{\infty} \Pi_{q}(p)$ and $\Pi_{q}(p) \subset$ $\Pi_{q+1}(p), q \in \mathbb{N}$. Therefore for each $p \in \mathbb{N}$ there is $q=\varrho(p)$ such that $\sup \left\{\pi(q): \pi \in \Pi_{q}(p)\right\}=\infty$. Now we fix an arbitrary $r \in \mathbb{N}$ and apply (8) with $q=\varrho(p)$ and $\pi \in \Pi_{q}(p)$ such that $\pi(q) \geq r$. Taking into account that

$$
|R|_{l, \pi(l)} \leq \begin{cases}|R|_{q, p} & \text { if } 1 \leq l \leq q, \\ |R|_{\mu(r), r} & \text { if } q<l \leq \mu(r),\end{cases}
$$

and that the same holds for $S$, we derive from (8) that

$$
|T|_{r, \varrho(p)} \leq \mu(r) \max \left\{|R|_{\varrho(p), p},|R|_{\mu(r), r}\right\} \cdot \max \left\{|S|_{\varrho(p), p},|S|_{\mu(r), r}\right\} .
$$

Hence one can easily conclude that there are $\tau \in \mathbb{N}^{\mathbb{N}}$ and $C=C(p, r)$ such that (7) holds. Thus $(F, G, E) \in \overline{\mathcal{S F}}$.

In particular, if $F=G$ or $G=E$, we get the following

Corollary 8. Let $E$ and $F$ be Fréchet spaces. Then $(E, F) \in \mathcal{B}$ implies $(F, E) \in \overline{\mathcal{S}}$.

This is a generalization of Proposition 3.4 from [5], where the case of Köthe spaces was considered (for Köthe spaces the conditions $\mathcal{S}$ and $\overline{\mathcal{S}}$ coincide): basically, our proof of Proposition 7 is a generalized direct version of the proof ad absurdum from [5]). 
Now we compare the conditions $\mathcal{S}$ and $\overline{\mathcal{S}}$ with their factorized versions.

Proposition 9. Let $E, G$, and $F$ be arbitrary Fréchet spaces. If the couple $(F, E)$ satisfies $\overline{\mathcal{S}}($ or $\mathcal{S})$, then the triple $(F, G, E)$ satisfies $\overline{\mathcal{S} \mathcal{F}}$ (respectively, $\mathcal{S F})$.

Proof. Because of complete similarity we consider only the case $\overline{\mathcal{S}}$. Suppose that $(F, E) \in \overline{\mathcal{S}}$. Then there is a function $\tau: \mathbb{N} \rightarrow \mathbb{N}$ such that for each $T \in L(E, F)$ the estimate

$$
|T|_{r, \tau(p)} \leq C \max \left\{|T|_{\tau(p), p},|T|_{\tau(r), r}\right\}
$$

holds for all $p, r \in \mathbb{N}$ with some constant $C=C(p, r)$. Without loss of generality we assume $\tau(p) \geq p$ for every $p \in \mathbb{N}$. Using now the evident estimate

$$
|T|_{\tau(p), p} \leq|S|_{p, p} \cdot|R|_{\tau(p), p} \leq|S|_{\tau(p), p} \cdot|R|_{\tau(p), p}, \quad p \in \mathbb{N},
$$

for any operator $T=R S$, we obtain the estimate (7), which means that $(F, G, E) \in \overline{\mathcal{S F}}$.

The following example shows that $\mathcal{S} \mathcal{F}$ is strictly weaker than $\mathcal{S}$. Here we use the notation $\Lambda_{\alpha}(a):=K\left(\exp \left(\alpha_{p} a_{i}\right)\right)$ with $\alpha_{p} \uparrow \alpha \leq \infty, a=\left(a_{i}\right)$.

ExAmPle. Let $a=\left(a_{i}\right)$ be a positive sequence increasing to infinity. Since $\left(\Lambda_{1}(a), \Lambda_{\infty}(a)\right) \in \mathcal{B}([14])$, we have $\left(\Lambda_{1}(a), \Lambda_{\infty}(a), \Lambda_{1}(a)\right) \in \mathcal{B F}$ trivially. Hence $\left(\Lambda_{1}(a), \Lambda_{\infty}(a), \Lambda_{1}(a)\right) \in \mathcal{S} \mathcal{F}$ by Proposition 7 . However $\left(\Lambda_{1}(a), \Lambda_{\infty}(a)\right) \notin \mathcal{S}$.

We conclude with a generalizaton of Djakov-Ramanujan's result ([2, Proposition 3]) in the context of factorization.

Theorem 10. Suppose $(\lambda(A), \lambda(B), \lambda(C)) \notin \mathcal{B F}$ and $(\lambda(C), \lambda(B), \lambda(A))$ $\in \mathcal{S} \mathcal{F}$. Then one of the pairs $(\lambda(A), \lambda(B))$ or $(\lambda(B), \lambda(C))$ has a common basic subspace.

Proof. By Theorem 1 there exist quasidiagonal operators $S \in Q_{\sigma}(A, B)$ and $R \in Q_{\varrho}(B, C)$ with $\sigma$ and $\varrho$ bijective such that $T=R S$ is unbounded. Without loss of generality we assume in what follows that all three operators are identity embeddings, since otherwise we can get this property by considering a new triple of Köthe spaces obtained from the original one by some permutations and normalizations of their canonical bases (note that the property $\mathcal{S} \mathcal{F}$ is preserved under such reconstruction). When applied to the above embeddings, the condition $\mathcal{S F}$ gives the following: there is a map $\tau: \mathbb{N} \rightarrow \mathbb{N}$ such that

$$
\frac{c_{i}^{r}}{a_{i}^{\tau(p)}} \leq C \max \left\{\frac{b_{i}^{\tau(p)}}{a_{i}^{p}}, \frac{b_{i}^{\tau(r)}}{a_{i}^{r}}\right\} \cdot \max \left\{\frac{c_{i}^{\tau(p)}}{b_{i}^{p}}, \frac{c_{i}^{\tau(r)}}{b_{i}^{r}}\right\}
$$

for all $(p, r, i) \in \mathbb{N}^{3}$ with some constant $C=C(p, r)$. 
It now suffices to prove that there is an infinite set $I \subset \mathbb{N}$ such that $\lambda(A)_{I}=\lambda(B)_{I}$ or $\lambda(B)_{I}=\lambda(C)_{I}$. Suppose that this assertion is false. Then for each infinite set $I \subset \mathbb{N}$ and $m \in \mathbb{N}$ there is $r \geq m$ such that

$$
\liminf _{i \in I} \frac{b_{i}^{\tau(r)}}{a_{i}^{r}}=\liminf _{i \in I} \frac{c_{i}^{\tau(r)}}{b_{i}^{r}}=0 .
$$

We define inductively the sets $N_{0} \supset N_{1} \supset \ldots$ by

$$
N_{0}:=\mathbb{N}, \quad N_{p}:=\left\{i \in N_{p-1}: \max \left\{\frac{b_{i}^{\tau(p)}}{a_{i}^{p}}, \frac{c_{i}^{\tau(p)}}{b_{i}^{p}}\right\} \geq 1\right\}, \quad p \in \mathbb{N},
$$

with $\tau$ from (10).

We claim that for each $p \in \mathbb{N}$ the embedding $T$ is unbounded on the basic subspace $X_{p}$ of $\lambda(A)$ spanned by $\left\{e_{i}: i \in N_{p-1} \backslash N_{p}\right\}$. If that is not so, then for each $q \in \mathbb{N}$ there is an infinite subset $I_{q} \subset N_{p-1} \backslash N_{p}$ and $m(q) \in \mathbb{N}$ with

$$
\lim _{i \in I_{q}} \frac{c_{i}^{m(q)}}{a_{i}^{q}}=\infty .
$$

For $I=I_{q}$ we find $r \geq m(q)$ such that (11) holds. Then there is an infinite set $J_{q} \subset I_{q}$ with

$$
\max \left\{\frac{c_{i}^{\tau(r)}}{b_{i}^{r}}, \frac{c_{i}^{\tau(r)}}{b_{i}^{r}}\right\}<1, \quad i \in J_{q} .
$$

On the other hand, by (12), we have

$$
\max \left\{\frac{c_{i}^{\tau(p)}}{b_{i}^{p}}, \frac{c_{i}^{\tau(p)}}{b_{i}^{p}}\right\}<1, \quad i \in I_{q} .
$$

Applying now (10) with $q=\tau(p)$ and $r$ chosen above and taking into account the estimates (14) and (15), we obtain

$$
\frac{c_{i}^{r}}{a_{i}^{q}} \leq C
$$

for all $i \in J_{q}$, which contradicts (13). This proves our claim that the embed$\operatorname{ding} T$ is bounded on each $X_{p}$. Hence, for every $p \in \mathbb{N}$, the operator $T$ must be unbounded on the basic subspace $Y_{p}$ generated by $\left\{e_{i}: i \in N_{p}\right\}$, which, in particular, implies that $N_{p}$ is an infinite set.

Now we construct a sequence $I=\left\{i_{p}\right\}$ so that $i_{p} \in N_{p}, i_{p+1} \neq i_{p}, p \in \mathbb{N}$. Then, due to (12), there is an infinite set $J \subset I$ such that at least one of the inequalities $a_{i}^{p} \leq b_{i}^{\varrho(p)}$ or $b_{i}^{p} \leq c_{i}^{\varrho(p)}$ holds for all $p \in \mathbb{N}$ and all $i \in J$ such that $i \geq p$, which contradicts the assumption (11). This completes the proof.

Acknowledgements. The authors would like to thank the referee for his/her suggestion how to simplify essentially the proof of Lemma 4. 
T. Terzioğlu et al.

\section{References}

[1] H. Apiola, Characterization of subspaces and quotients of nuclear $L_{f}(a, \infty)$-spaces, Compositio Math. 50 (1983), 65-81.

[2] P. B. Djakov and M. S. Ramanujan, Bounded and unbounded operators between Köthe spaces, Studia Math. 152 (2002), 11-31.

[3] M. M. Dragilev, Riesz classes and multiple regular bases, Teor. Funktsiü Funktsional. Anal. i Prilozhen. 15 (1972), 65-77 (in Russian).

[4] L. Frerick, A splitting condition for nuclear Fréchet spaces, in: Functional Analysis (Trier, 1994), de Gruyter, Berlin, 1996, 163-167.

[5] J. Krone and D. Vogt, The splitting relation for Köthe spaces, Math. Z. 190 (1985), $387-400$.

[6] Z. Nurlu, On basic sequences in some Köthe spaces and existence of non-compact operators, Ph.D. thesis, Clarkson College of Technology, Potsdam, NY, 1981.

[7] -, On pairs of Köthe spaces between which all operators are compact, Math. Nachr. 122 (1985), 277-287.

[8] Z. Nurlu and T. Terzioğlu, Consequences of the existence of a non-compact operator between nuclear Köthe spaces, Manuscripta Math. 47 (1984), 1-12.

[9] T. Terzioğlu and V. Zahariuta, Bounded factorization property for Fréchet spaces, Math. Nachr. 253 (2003), 1-11.

[10] D. Vogt, Frécheträume, zwischen denen jede stetige lineare Abbildung beschränkt ist, J. Reine Angew. Math. 345 (1983), 182-200.

[11] - On the functor $\operatorname{Ext}^{1}(E, F)$ for Fréchet spaces, Studia Math. 85 (1987), 163-197.

[12] - Regularity properties of (LF)-spaces, in: Progress in Functional Analysis, NorthHolland Math. Stud. 170, North-Holland, Amsterdam, 1992, 57-84.

[13] V. Zahariuta, On isomorphisms of Cartesian products of linear topological spaces, Funktsional. Anal. i Prilozhen. 4 (1970), no. 2, 87-88 (in Russian).

[14] - On the isomorphism of cartesian products of locally convex spaces, Studia Math. 46 (1973), 201-221.

T. Terzioğlu

Sabancı University

81474 Tuzla-Istanbul, Turkey

E-mail: tosun@sabanciuniv.edu.tr

M. Yurdakul

Department of Mathematics

Middle East Technical University

06531 Ankara, Turkey

E-mail: myur@metu.edu.tr
V. P. Zahariuta

Department of Mathematics Middle East Technical University

Faculty of Engineering and Natural Sciences

Sabancı University

81474 Tuzla-Istanbul, Turkey

E-mail: zaha@sabanciuniv.edu.tr

Received August 12, 2002

Revised version March 6, 2003 